\title{
Extending the usable Ka band spectrum for satellite communications: The CoRaSat project
}

\author{
Barry Evans ${ }^{1}$, Paul Thompson ${ }^{1}$, Eva Lagunas ${ }^{2}$ Shree Krishna Sharma ${ }^{2}$, \\ Daniele Tarchi ${ }^{3}$, and Vicenzo Riccardo Icolari ${ }^{3}$ \\ 1 University of Surrey, Guildford, GU2 7XH, UK, \\ \{b.evans, p.thompson\}@surrey.ac.uk, \\ 2 University of Luxembourg, Luxembourg \\ 3 University of Bologna, Italy \\ \{daniele.tarchi, vincenzo.icolari2\}@unibo.it
}

\begin{abstract}
Broadband access by satellite in Ka band will become constrained by spectrum availability. In this context, the EU FP7 project CoRaSat is examining the possible spectrum extension opportunities that could be exploited by a database or sensing approach in Ka band via the use of cognitive mechanisms. The database/sensing approach utilises spectrum sharing scenarios between Fixed Satellite Services (FSS), Fixed Services (FS) and Broadcast Satellite Service (BSS) feeder links are considered. Data bases and spectrum sensing have been evaluated to determine white spaces across the shared spectrum for several EU countries. Resource allocation schemes are investigated to place the carriers in the white spaces so as to maximize the throughput of the system. A multibeam satellite system model has been used to demonstrate the capacity gains that can be achieved by using the cognitive schemes. The overall system is being demonstrated in a laboratory trial.
\end{abstract}

Key words: Satellite/terrestrial, spectrum sharing, data bases, spectrum sensing, resource allocation.

\section{INTRODUCTION}

The demand for higher rate and reliable broadband communications is accelerating all over the world. Within Europe the Digital Agenda sets a target for universal broadband coverage of at least 30 Mbps across the whole of Europe by 2020 and $100 \mathrm{Mbps}$ to at least $50 \%$ of the households [1]. Fixed connections and cellular cannot alone meet this target, particularly in the rural and remote areas but also in some black spots across the coverage. In these latter regions satellite broadband delivery is the only practical answer as satellite will cover the whole territory. Some recent studies of the roll out of broadband have shown that up to $50 \%$ of households in some regions will only have satellite available as a means of accessing broadband and thus 5-10 million households are potential satellite customers [2]. Current $\mathrm{Ku}$ band satellites do not have the capacity to deliver such services at a cost per bit that makes a business case and thus the satellite community has turned to High Throughput Satellites (HTS) operating 
at Ka band and above. Examples of early HTS Ka band satellites dedicated to such services are Eutelsats KaSat [3] and VIASAT 1 [4]. These satellites employ multiple (around 100) beams using four fold frequency reuse over the coverage area to achieve capacity of the order of $100 \mathrm{Gbps}$ per satellite. The latter is limited by the exclusive spectrum available to satellite (FSS) of $500 \mathrm{MHz}$ in both the up and downlinks and this limits the feasible user rates to 10-20 Mbps. Thus looking ahead to the increased user demands we have to look to larger satellites (maybe up to a Terabit/s $[5,6]$ ) and to more spectrum. Moving up to Q/V bands has already been suggested for feeder links but for user terminals the additional expense is not considered desirable so we return to the problem of getting more usable spectrum at Ka band.

The Ka-Band exclusive bands for satellite are 19.7 to $20.2 \mathrm{GHz}$ in the downlink and 29.5 to $30 \mathrm{GHz}$ on the uplink. In these bands FSS terminals can operate in an uncoordinated manner, which means that they do not have to apply for and be granted a licence by the national regulators, provided they meet set performance characteristics. The issue in other parts of the Ka band is that the spectrum is allocated, not just to FSS but also to fixed links (FS) and to BSS (uplinks for broadcast satellites) as well as mobile services (MS). This spectrum is allocated by the ITU in three regions of the world as shown in Table 1 for Ka band (Europe is Region 1). In these so-called shared bands the different services need to co-exist. Within Europe the CEPT [7] have adopted decisions that expand those of the ITU and produce tighter regulation as follows;

- 17.3-17.7 GHz: the BSS feeder links are determined as the incumbent links but uncoordinated FSS links are also permitted in this band.

- 17.7-19.7 GHz: FS links are considered incumbent but FSS terminals may be deployed anywhere but without right of protection.

- 27.5-29.5 GHz: CEPT provide a segmentation of the band between FSS and FS portions. Within each segment there is a specified incumbent but for instance FSS terminals can operate in FS portions provided they do not interfere with the incumbent FS.

The work reported in this paper has been conducted within the EU FP7 project CoRaSat $[8,9,10,11]$ which examines ways in which FSS satellite terminals in the Ka band can co-exist with FS and BSS links given the regulatory regime discussed above. Specifically, a database approach for such coexistence schemes is investigated and demonstrated to exploit the frequency sharing opportunities for uncoordinated FSS terminals and verify its applicability. The aim is to show that future satellite systems can access additional spectrum beyond the exclusive bands that is needed to deliver cost effective broadband services.

\section{SCENARIOS AND DATABASE APPROACH}

Within the CoRaSat project, three scenarios have been investigated that reflect the three spectrum components detailed in the previous section. In Fig.1 we illus-

trate the interference paths in these scenarios. Two of the scenarios are downlink 
for the FSS; scenario A, 17.3-17.7 GHz where the potential interference is from BSS uplinks and scenario B, 17.7-19.7 GHz where the potential interference is from incumbent FS transmitters. In both of these cases the FSS is permitted to operate but is not protected by the regulatory regime and thus it is important to ascertain the level of the interference and its affect on the FSS received signal. The third scenario C, is in the transmit band of the FSS from 27.5-29.5 GHz and the interference is from the FSS transmitting earth station into the FS receivers which are protected. The latter is more critical in that we need to demonstrate that the FSS does not contravene interference limits imposed by the regulatory regime. The forward link, e.g. the downlink can be considered more important as it carries more capacity and in addition to this, operation in the downlink bands do not require regulatory changes but merely reassurance to the FSS users that the services need not be impaired. The calculation of interference can be per-

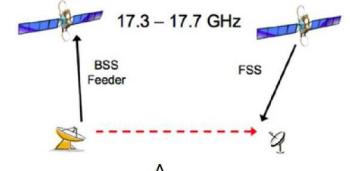

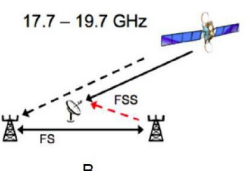

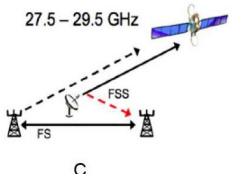

Fig. 1. Scenarios in CoRaSat.

formed if the corresponding accurate database has been obtained, which includes the characteristics and locations of the potential interferers, by using accurate models of the equipment, propagation and the path details. Similar ideas have been employed in TVWS systems [12] to allow UHF frequencies to be used in the gaps between TV transmission regions. For scenario A the number of BSS uplinks in Europe is small and thus a database system is similar in magnitude to that of TVWS. However for scenario B and C the number of FS links runs into the tens of thousands and the database is much more complex. The data on the positions and the characteristics of the BSS and FS are generally held by national regulators and these need to be available for a database system to work.

The information of a real interferer database is interfaced to an interference modeling engine which uses ITU- Recommendation P.452-15 [13] procedures plus terrain databases. This is the latest version of this ITU Recommendation that contains a prediction method for the evaluation of path loss between stations. ITU-R P.452-15 includes all the propagation effects on the surface of the Earth at frequencies from $0.1 \mathrm{GHz}$ to $50 \mathrm{GHz}$. In addition, other factors which affect interference calculation, such as terrain height, bandwidth overlapping are also considered in the proposed database approach, which is illustrated in Fig. 2. The typical interference threshold we determine is based on the long term interference which can be expected to be present for at least $20 \%$ of the average year and it is set at $10 \mathrm{~dB}$ below the noise floor. The interference thresholds 
Table 1. ITU-R Table of allocation

\begin{tabular}{|c|c|c|c|}
\hline Frequency bands & ITU Region 1 & ITU Region 2 & ITU Region 3 \\
\hline $\begin{array}{l}\text { 17.3-17.7 GHz } \\
\text { (Scenario A) }\end{array}$ & $\begin{array}{c}\text { FSS } \\
\text { (space-Earth) } \\
\text { BSS (feeder } \\
\text { links) } \\
\text { Radiolocation }\end{array}$ & $\begin{array}{c}\text { FSS } \\
\text { (space-Earth) } \\
\text { BSS (feeder } \\
\text { links) } \\
\text { Radiolocation }\end{array}$ & $\begin{array}{c}\text { FSS (space } \\
\text { Earth)BSS } \\
\text { (feeder links) } \\
\text { Radiolocation }\end{array}$ \\
\hline $\begin{array}{c}17.7-19.7 \mathrm{GHz} \\
\text { (Scenario B) }\end{array}$ & $\begin{array}{c}\text { FSS } \\
\text { (space-Earth) } \\
\text { BSS (feeder links } \\
\text { up 18.1 GHz) FS }\end{array}$ & $\begin{array}{c}\text { FSS } \\
\text { (space-Earth) FS }\end{array}$ & $\begin{array}{c}\text { FSS } \\
\text { (space-Earth) } \\
\text { BSS (feeder links } \\
\text { up 18.1 GHz) FS }\end{array}$ \\
\hline $\begin{array}{c}27.5-29.5 \mathrm{GHz} \\
\text { (Scenario } \mathrm{C} \text { ) }\end{array}$ & $\begin{array}{c}\text { FSS } \\
\text { (Earth-space) FS } \\
\text { MS (Mobile } \\
\text { Services) }\end{array}$ & $\begin{array}{c}\text { FSS } \\
\text { (Earth-space) FS } \\
\text { MS }\end{array}$ & $\begin{array}{c}\text { FSS } \\
\text { (Earth-space) FS } \\
\text { MS }\end{array}$ \\
\hline
\end{tabular}

for FSS reception and for FS reception are therefore $-154 \mathrm{dBW} / \mathrm{MHz}$ and -146 $\mathrm{dBW} / \mathrm{MHz}$, respectively as given in [14] and [15].

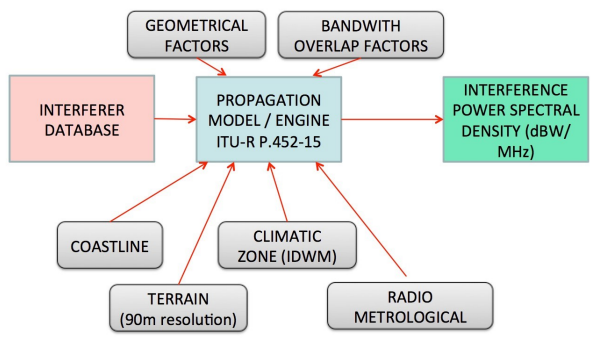

Fig. 2. Interference modelling by ITU-R P.452-15.

Having determined the interference level at the FSS (in scenarios A or B) it can be compared with the regulatory threshold. The action is then taken in the resource allocation at the gateway where a new carrier can be assigned either in another part of the shared band where interference is acceptable or in the exclusive band. For scenario $\mathrm{C}$ the situation is different as the interference is caused by the FSS into the FS. Here the database is used to calculate the maximum permissible power that can be transmitted from the FSS in the vicinity in order to retain the threshold condition at the FS receivers

Scenario A

A UK BSS database made available for this study is used for scenario A and contains 442 carriers from a total of 31 BSS uplink earth stations at 8 physical sites, to 12 different satellites. The number of carriers of each BSS earth station ranges from 1 to 42 . The carriers span the range $17.3 \mathrm{GHz}$ to $18.35 \mathrm{GHz}$. The 


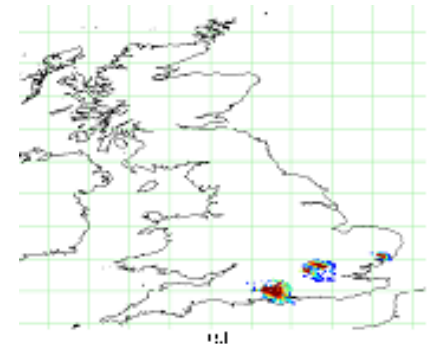

(a)

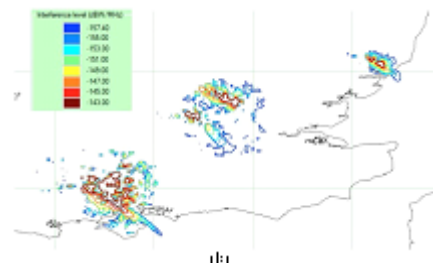

(b)

Fig. 3. Example of cognitive zones for the sub-band $1(17.3-17.34 \mathrm{GHz})$ based on full ITU model.

bandwidths of the carriers that belong to the same BSS earth station are the same while those that belong to different earth stations might be different and are typically $26 \mathrm{MHz}, 33 \mathrm{MHz}, 36 \mathrm{MHz}$ or $66 \mathrm{MHz}$. The EIRP of these earth station antennas ranges from $69 \mathrm{dBW}-84 \mathrm{dBW}$ and all antenna radiation patterns are as defined in ITU-Recommendation S.465 [16] or S.580 [17]. Using the BSS database, area analysis for scenario A in the UK is provided to investigate how much area would be affected by interference from the BSS feeder links. The band of interest is split into $10 \times 40 \mathrm{MHz}$ sub-bands (SB1/SB10) and the analysis is then conducted in each sub-band to determine the area of the contours at different cognitive zone thresholds. These mirror the usual $40 \mathrm{MHz}$ channel spacing adopted for BSS satellites. Area analysis is based on BSS database with the full ITU-R P.452-15 model employing the terrain and climatic zones and the FSS terminal evaluated points to a satellite at 53 degrees E longitude. The results are for long term interference (normally 20\%). One example of affected area at difference cognitive zone thresholds is shown in Fig. 3, which represents SB1. It was found that in general across the sub-bands at a $-155 \mathrm{dBW} / \mathrm{MHz}$ threshold less than for $2 \%$ of the area of the UK is affected by BSS feeder links and thus more than $98 \%$ of the area of the UK can be used by an FSS terminal without the need for any further action We have performed the same analysis for Luxembourg with very similar results and as the UK is the most dense BSS case we would expect the results to be similar or better in other countries.

Scenario B

FS data bases at $18 \mathrm{GHz}(17.7$ to $19.7 \mathrm{GHz})$ were used to evaluate the interference. An up to date FS data base was made available to this project by Ofcom UK. This data base for the UK FS in the band 17.7 to $19.7 \mathrm{GHz}$ contains 15,036 carrier records. A French data base has also been examined at $18 \mathrm{GHz}$ and is based on the latest ITU terrestrial services BR IFIC database [18], which contains 16,136 carrier records. Similarly data for Hungary and Slovenia has been obtained from the same source and these contain 2,402 and 1,237 carrier records respectively. Data at $18 \mathrm{GHz}$ for Poland was obtained which is more up to date than the BR IFIC data and contains 8,323 carrier records. An example of the spectrum occupancy for an FSS terminal placed in the SE of the UK is 


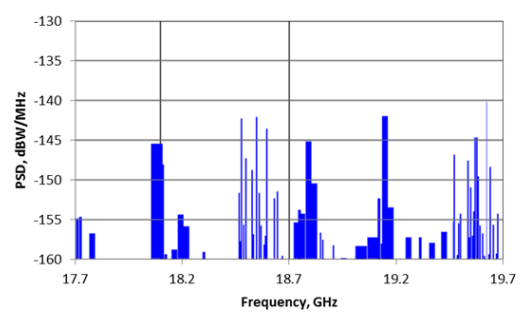

(a)

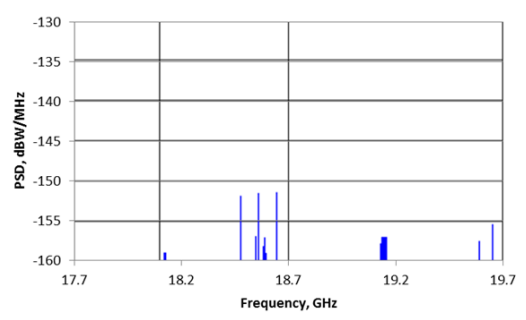

(b)

Fig. 4. Channel occupancy at an FSS point in SE UK for (a)Free space loss model and (b) Full ITU model.

shown in Fig. 4. Here the calculation based on free space loss only and the full ITU model clearly shows the necessity for inclusion of the terrain effects. It is also clear that white spaces exist for FSS carriers at this location. However the actual position of the white spaces varies with location and thus the data base can be used together with the resource allocation scheme to place the carriers appropriately to the FSS terminals. By analysing the interference results for five data bases for different countries it is possible to get an increased insight into the situation. A CDF is shown for the total occupied bandwidth of the FS interferers at a point over the regions of interest in Fig. 5. This demonstrates that for the majority of locations a large percentage of the $2 \mathrm{GHz}$ spectrum is available for FSS use in all of the countries examined.

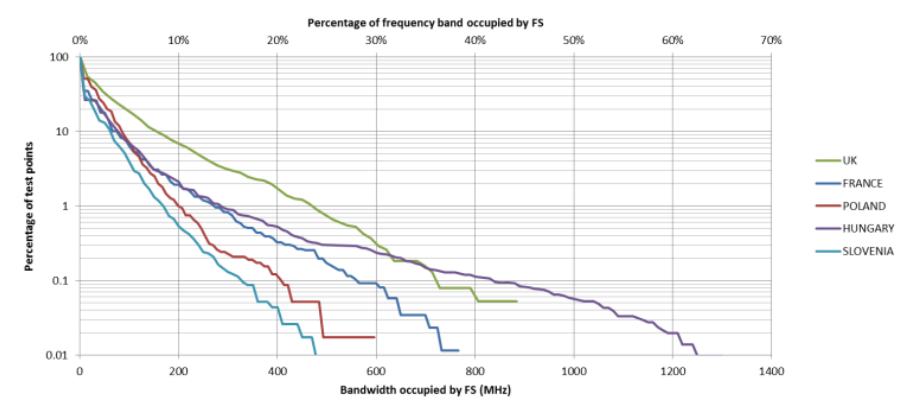

Fig. 5. CDF of FS bandwidth available for threshold of -154dBW/MHz.

\section{Spectrum Sensing}

A database approach, although very efficient, requires knowledge on BSS and FS links, which might be confidential for some countries. Moreover, even in 
countries where such information is available, the database approach does not allow to adapt to short-term variations in spectrum occupancy.

In this scenario, coexistence between FSS cognitive systems and incumbent systems is thus limited by the interference generated from the latter towards the FSS terminal. In particular, a significant amount of aggregate interference may occur at a given FSS terminal due to the side-lobes of the receiving antenna pattern. CR techniques can thus be employed to foster the coexistence between FSS DL and incumbent links, as shown in the following sections. In the following, it is assumed that the receiving chain at the cognitive terminal is used for both sensing and secondary transmissions.

Among several Spectrum Sensing techniques [19], we will firstly focus on energy detection (ED) that is assessed in the considered scenario. Simulation results show that CR-based satellite systems can significantly improve spectrum utilization, which would enable the integration between terrestrial and satellite networks, as well as provide additional spectrum for both systems.

Spectrum sensing (SS) aims at detecting the incumbent user signal by scanning a selected frequency band B $[19,20]$. It refers to the detection of an unknown or partially known signal, and a trade-off between the probability of false alarm (Pf ) and the probability of detection (Pd) is necessary for achieving an accurate degree of certainty in such detection. SS techniques can be modeled as a binary hypothesis test problem, comparing a statistical metric with a given threshold.

An energy detector aims at detecting the presence of incumbent signals based on the energy estimated at the antenna input of the cognitive terminal [21, 22]. It is a blind detection technique, as it does not require a-priori knowledge on the incumbent signal, and therefore has a general applicability in CR-based systems. However, it is highly susceptible to Signal-to-Noise Ratio (SNR) wall problem, that prevents from achieving the desired target probabilities Pd or Pf , as the uncertainty in noise power estimation, $\mathrm{N}$, can easily erroneously trigger the detection $[23,24]$.

As an example, in Fig. 6 the comparison between a real interference map, obtained by comparing the real interference values with a certain threshold $(\mathrm{I} / \mathrm{N})=-$ $10 \mathrm{~dB}$, and the interference map obtained through the use of an Energy Detector are shown. In Table 2, the parameters used for obtaining the numerical results of the ED map are listed.
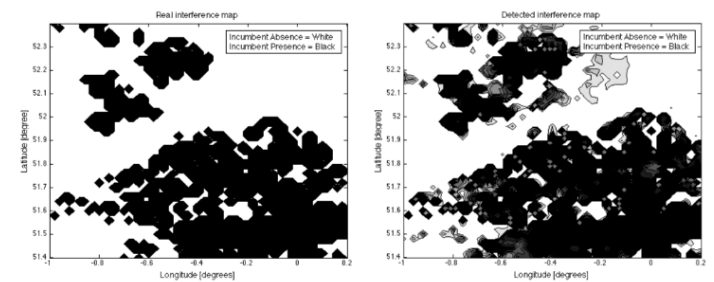

Fig. 6. Comparison between a real interference map and the detected interference map by exploiting the ED. 
Table 2. Simulation parameters for the ED.

\begin{tabular}{|c|c|}
\hline Frequency [GHz] & 17.634 \\
\hline Bandwidth [MHz] & 36 \\
\hline FSS Terminal Latitude & From $51.4 \mathrm{~N}$ to $52.4 \mathrm{~N}$ \\
\hline FSS Terminal Longitude & From $1.0 \mathrm{~W}$ to $0.2 \mathrm{E}$ \\
\hline FSS Satellite Longitude & $53 \mathrm{E}$ \\
\hline Probability & Pfa $=0.1$ \\
\hline Sensing Time [us] & 20 \\
\hline Noise uncertainty [dB] & 0 \\
\hline Number of simulation & 10000 \\
\hline
\end{tabular}

Within the proposed scenarios, a Signal-to-Interference plus Noise Ratio (SINR) estimation algorithm has been also proposed. Among different interference estimators available in the literature [25], we rely on the Data Aided SNORE (DA-SNORE) algorithm described in [26]. It is assumed that the cognitive Earth terminal is equipped with a receiving chain able to scan all frequencies of interest with a sensing sub-band equal to $36 \mathrm{MHz}$, which is the typical bandwidth of DVB-S2 and DVB-S2x standards [27], used by the cognitive satellite system. The algorithm, described in [28], is based on the knowledge of the pilot blocks of the DVB-S2 standard. It is worthwhile highlighting that, as the pilot blocks are the same for both Scenario A and B, the algorithm can be applied with no modification to either of them.

Focusing on Scenario A, the $400 \mathrm{MHz}$ band is split into 11 sub-bands, and on each sub-band the DA-SNORE algorithm is applied to determine the interference level received from BSS feeder links. As the incumbent spectrum utilization is almost constant in time, the sensing operation can be performed with a relatively low duty cycle and when no data transmission is required, so as to lower the computational load. The information gathered during this initial sensing phase can then be reported to the Network Control Center (NCC), which allocates to each user the most reliable sub-band.

The performance of the SINR estimation algorithm has been compared to data extracted from databases. In particular, the potential geographical reuse factor of a specific carrier as a function of the relative location between interferer and interfered terminals has been performed. As an example, Fig. 7 provides the SINR values obtained from the database over a specific geographic area and compares them to the values estimated through the DA-SNORE algorithm. For obtaining the comparison, the same parameters listed in Table 2 have been used, with, additionally, a number of pilots equal to 10 and the SNR value at the DASNORE based interference estimator antenna input equal to $4 \mathrm{~dB}$.

The estimated values excellently match the SINR values obtained from the database, and thus the DA-SNORE algorithm provides a valuable solution for spectrum awareness either to complement the information stored in databases or to provide the spectrum occupancy when databases are not available. 

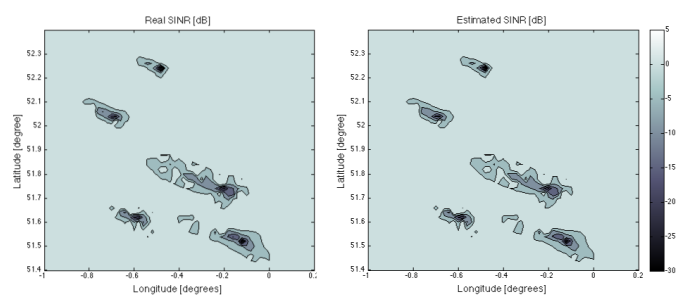

Fig. 7. Comparison between Real SINR and Estimated SINR by exploiting the proposed DA-SNORE based algorithm.

\section{Resource allocation}

After obtaining the spectrum awareness, the available resources need to be allocated among the cognitive terminals. In this section, we provide a numerical evaluation of the resource management techniques presented in the context of [8], which aim at optimizing the allocation of available resources, while employing interference management techniques. Given the similarities between Scenario A and Scenario B, dynamic carrier assignment (CA) techniques based on Signalto-Interference-plus-Noise Ratio (SINR) are applied in both cases. In Scenario $\mathrm{A}$ and $\mathrm{B}$, beamforming $(\mathrm{BF})$ will be considered together with $\mathrm{CA}$ in order to mitigate the received interference and enlarge the cognitive zones presented in Section II. Essentially, the level of FS interference at the carrier level is firstly determined based on the available information of FS databases. Having determined the interference level and using the signal level obtained from the FSS system analysis, the SINR is computed for all the FSS terminals considering all the carrier frequencies. Subsequently, we apply BF only in the FSS terminals which suffer excessive interference. Next, the improved SINR is fed to the CA module in order to assign each user to a carrier so as to maximize the total sum-rate of the system.

Here, we present results for Scenario B. Scenario A, as indicated in Section II, is expected to provide higher gains due to a few number of BSS feeder links. For Scenario B evaluation, we consider the country France and the selected beams are depicted in Fig. 8. These beams have been selected based on the potential FS interference receivers and the final results are obtained based on the weighting factor provided in Table 3 .

The results shown in this section were obtained after 50 Monte Carlo runs, in which the locations of the FSS terminals were selected uniformly at random for each realization within the considered beam coverage according to the population density database produced by NASA Socioeconomic Data and Applications Center (SEDAC) [29]. The considered system parameters are summarized in Table 4.

The evaluation is made in the following cases:

- Case 1: Exclusive band only: In this case, the SINRs and user rates are calculated using only exclusive carriers. 
Table 3. Selected beams for Scenario B.

\begin{tabular}{|c|c|c|}
\hline & FS links & Weighting factor \\
\hline 1 & 1522 & 0.038462 \\
\hline 2 & 1681 & 0.076923 \\
\hline 3 & 635 & 0.5 \\
\hline 4 & 906 & 0.26923 \\
\hline 5 & 1220 & 0.11538 \\
\hline
\end{tabular}

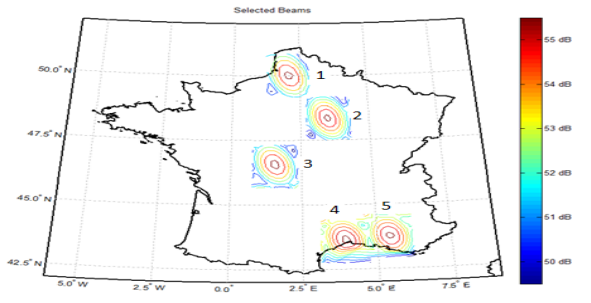

Fig. 8. Selected beams according to FS antenna density.

Table 4. Simulation Parameters for Scenario B.

\begin{tabular}{|c|c|}
\hline Simulation Parameter & Value \\
\hline Carrier Bandwidth & $63.4 \mathrm{MHz}$ \\
\hline Shared Band & $17.7-19.7 \mathrm{GHz}(32$ carriers $)$ \\
\hline Exclusive Band & $19.7-20.2 \mathrm{GHz}(8$ carriers $)$ \\
\hline Satellite & $13^{\circ} \mathrm{E}$ \\
\hline EIRP satellite & $65 \mathrm{dBW}$ \\
\hline Re-use Pattern & 4 color \\
\hline FSS antenna gain $(\max )$ & $42.1 \mathrm{~dB}$ \\
\hline FSS Rx noise temperature & $262 \mathrm{~K}$ \\
\hline FSS terminal height & $15 \mathrm{~m}$ \\
\hline LNBs at FSS terminal & 3 \\
\hline
\end{tabular}

- Case 2: Shared plus exclusive band w/o FS presence: In this case, the SNRs and user rates are calculated considering both shared and exclusive carriers, but without considering the FS system.

- Case 3: Shared plus exclusive bands w/ FS presence: In this case, the SINRs and user rates are calculated considering both shared and exclusive carriers, and considering the FS system.

The results of the five evaluated beams are shown in Table 5 in terms of per beam throughput (Mbps). The methodology followed for throughput evaluation is based on [30] and the employed carrier allocation and beamforming methods have been discussed in [30]. From Table 5, it can be noted that the throughput values significantly differ across the considered beams even for the case of exclusive only case, which is due to different beam gains and Carrier-to-Interference $(\mathrm{C} / \mathrm{I})$ values over these beams. The main conclusion that can be extracted from Table 5 is that that the throughput per beam improvement obtained with the proposed $\mathrm{CA}$ and $\mathrm{BF}$ techniques is $405.92 \%$. What is most important is that using the proposed $\mathrm{CA}$ and $\mathrm{BF}$ we can achieve similar average throughput as if there were no FS system. 
Table 5. Per beam throughput (Mbps) for Scenario B.

\begin{tabular}{|c|c|c|c|c|c|c|}
\hline \multirow{2}{*}{ Beam No. } & \multicolumn{2}{|c|}{ Case 1 } & \multicolumn{2}{c|}{ Case 2 } & \multicolumn{2}{c|}{ Case 3 } \\
\cline { 2 - 7 } & w/ o CA & w/ CA & w/o CA & w/CA & w/o CA & w/ CA+BF \\
\hline 1 & 675.10 & 675.42 & 3414.17 & 3419.78 & 3413.73 & 3468.05 \\
\hline 2 & 679.13 & 679.49 & 3404.98 & 3410.56 & 3404.25 & 3457.66 \\
\hline 3 & 660.42 & 660.72 & 3304.87 & 3309.07 & 3304.11 & 3331.52 \\
\hline 4 & 725.76 & 725.95 & 3641.67 & 3646.28 & 3640.18 & 3661.03 \\
\hline 5 & 718.47 & 718.85 & 3626.94 & 3646.07 & 3623.62 & 3659.71 \\
\hline Average & 686.56 & 686.84 & 3444.97 & 3451.16 & 3443.74 & 3473.46 \\
\hline
\end{tabular}

The applicability of resource allocation techniques in Scenario C was discussed in [31]. Here, we summarize the results obtained for Scenario C in Finland, from which a reliable FS Database obtained from the national regulator is used. Again, we select the most representative beams in terms of FS density. The beams are shown in Fig. 9 and Table 6 summarizes the details.

Table 6. Per beam throughput (Mbps) for Scenario B.

\begin{tabular}{|c|c|c|}
\hline & Number of Rx FS & Weight \\
\hline 1 & 32 & 0.222 \\
\hline 2 & 902 & 0.111 \\
\hline 3 & 6 & 0.667 \\
\hline
\end{tabular}

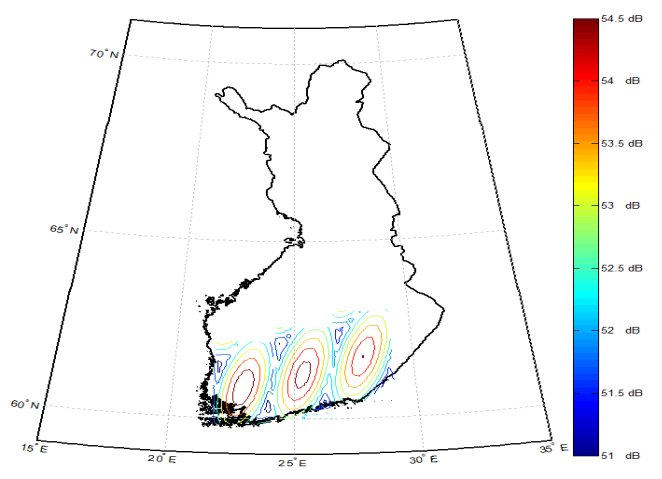

Fig. 9. Selected beams according to FS antenna density.

It can be noted that in Scenario $\mathrm{C}$, the cognitive transmitters create interference to the FS system and, thus, not only the carriers have to be optimally assigned but also the transmit power of the cognitive FSS terminal devices has to be adjusted so that the interference at each of the FS stations is kept below the given threshold. As we did for Scenario B, 50 Monte Carlo runs were averaged in which the FSS terminal locations were determined based on population data. A summary of the most relevant parameters and the FSS link budget details are presented in Table 7 .

The results of the three evaluated beams are shown in Table 8. It is important to keep in mind that with the proposed RA we ensure that we never violate the 
Table 7. Simulation Parameters for Scenario C.

\begin{tabular}{|c|c|}
\hline Simulation Parameter & Value \\
\hline Carrier Bandwidth & $7 \mathrm{MHz}$ \\
\hline Shared Band & $27.5-29.5 \mathrm{GHz}(285$ carriers $)$ \\
\hline Exclusive Band & $29.5-30 \mathrm{GHz}(71$ carriers $)$ \\
\hline Satellite & $13 \mathrm{E}$ \\
\hline EIRP & $50 \mathrm{dBW}$ \\
\hline Re-use Pattern & 4 color \\
\hline FSS antenna gain (max) & $42.1 \mathrm{~dB}$ \\
\hline$[\mathrm{G} / \mathrm{T}]$ (max) & $29.3 \mathrm{~dB} / \mathrm{k}$ \\
\hline FSS terminal height & $15 \mathrm{~m}$ \\
\hline
\end{tabular}

FS interference threshold. On average, the proposed RA provides $400 \%$ gain over the exclusive only band, which coincides with the best results that can be achieved in this particular scenario.

Table 8. Per beam throughput (Mbps) for Scenario C.

\begin{tabular}{|c|c|c|c|c|c|c|}
\hline \multirow{2}{*}{ Beam No. } & \multicolumn{2}{|c|}{ Case 1 } & \multicolumn{2}{c|}{ Case 2 } & \multicolumn{2}{c|}{ Case 3 } \\
\cline { 2 - 7 } & w/ o RA & w/ RA & w/o RA & w/RA & w/o RA & w/ RA \\
\hline 1 & 651.96 & 651.96 & 3258.19 & 3258.33 & 3253.60 & 3258.33 \\
\hline 2 & 683.53 & 683.53 & 3421.73 & 3422.31 & 3252.67 & 3422.31 \\
\hline 3 & 691.87 & 691.87 & 3449.44 & 3449.94 & 3449.30 & 3449.94 \\
\hline Average & 682.08 & 682.08 & 3403.91 & 3404.34 & 3384.03 & 3404.34 \\
\hline
\end{tabular}

\section{Conclusions}

It has been demonstrated that a data base system will allow frequency sharing in the 17.3 to $19.7 \mathrm{GHz}$ down link bands and in the 27.5 to $29.5 \mathrm{GHz}$ uplink band between satellite FSS and fixed FS links. Significant additional spectrum in the shared bands is available to satellite FSS and only small areas across Europe would need to adopt additional interference mitigation. Using a carrier resource allocation scheme at the satellite gateway it has been demonstrated that up to four times capacity gains can be achieved over the use of the exclusive band only. It has also been shown that spectrum sensing at the satellite terminals is feasible and can be used where data base information is not available or to augment and hence improve the data base. 


\section{ACKNOWLEDGEMENT}

The authors would like to acknowledge the EU FP7 project CoRaSat which has supported the work herein and in particular the inputs from industrial partners, SES,TAS and Newtec.

\section{References}

1. A Digital Agenda for Europe, FCC 02-155, European Commission COM 245, Brussels, Tech. Rep., 2010.

2. EU FP7 Project BATS, Vodafone Chair, Available: http://www.batsproject.eu/.

3. H. Fenech, E. Lance, and M. Kalama, KA-SAT and the way forward, Ka-Band Conference, Palermo, Italy., Tech. Rep., 2011.

4. Highest-capacity communications satellite, http://www.guinnessworldrecords.com/ records-1/ highest-capacity- communications-satellite/.

5. P. Thompson, B. Evans, L. Castenet, M. Bousquet, and T. Mathiopoulos, Concepts and technologies for a terabit/s satellite, in Proceedings of SPACOMM-2011 (best paper award in 2011), April 2011, Budapest, Hungary.

6. A. Kyrgiazos, B. Evans, P. Thompson, P. T. Mathiopoulos, and S. Pa- paharalabos, A terabit/second satellite system for european broadband access: a feasibility study, International Journal of Satellite Communi- cations and Networking, vol. 32, no. 2, 2014, pp. 6392.

7. The European conference of postal and telecommunications adminis- trations, avaliable: http://www.cept.org/cept.

8. EU FP7 Project CoRaSat, available: http://www.ict-corasat.eu.

9. K. Liolis, G. Schlueter, J. Krause, F. Zimmer, L. Combelles, J. Grotz, S. Chatzinotas, B. Evans, A. Guidotti, D. Tarchi, and A. Vanelli-Coralli, Cognitive radio scenarios for satellite communications: The corasat approach, in Future Network and Mobile Summit (FutureNetworkSum- mit), 2013, July 2013, pp. 110.

10. S. Maleki, S. Chatzinotas, B. Evans, K. Liolis, J. Grotz, A. Vanelli- Coralli, and N. Chuberre, Cognitive spectrum utilization in ka band multibeam satellite communications, IEEE Communication Magazine, accepted for publish.

11. Cognitive radio techniques for satellite communications operating in Ka band, Tech. Rep., ETSI System Reference document, available: http://webapp.etsi.org.

12. Standardization of TV white space systems, available: http://www.ictcrsi.eu/index.php/standardization-streams/tv-white-spaces.

13. Recommendation P.452-15: Prediction procedure for the evaluation of interference between stations on the surface of the earth at frequencies above about $0.1 \mathrm{GHz}$, International Telecommunication Union, Tech. Rep., 2013.

14. Methods for the determination of the coordination area around an earth station in frequency bands between $100 \mathrm{MHz}$ and $105 \mathrm{GHz}$, ITU Radio Regulation Appendix 7, International Telecommunication Union, Tech. Rep., 2012.

15. Recommendation F.758-5: System parameters and considerations in the development of criteria for sharing or compatibility between digital fixed wireless systems in the fixed service and systems in other services and other sources of interference, International Telecommunication Union, Tech. Rep., 2012.

16. Recommendation ITU-R S.465: Reference radiation pattern for earth station antennas in the fixed- satellite service for use in coordination and interference assessment in the frequency range from 2 to $31 \mathrm{GHz}$, International Telecommunication Union, Tech. Rep., 2010. 
17. Recommendation ITU-R S.580: Radiation diagrams for use as design objectives for antennas of earth stations operating with geostationary satellites, International Telecommunication Union, Tech. Rep., 2004.

18. ITU-R Terrestrial BRIFIC, available: http://www.itu.int/ITU$\mathrm{R} /$ index.asp?category $=$ terrestrial\&rlink=terrestrial-\%brific\&lang $=\mathrm{en}$.

19. S. Haykin, Cognitive radio: brain-empowered wireless communications, IEEE JSAC, vol. 23, no. 2, pp. 201220, Feb. 2005.

20. E. Hossain, D. Niyato, and Z. Han, Dynamic Spectrum Access and Management in Cognitive Radio Networks, Cambridge University Press, Cambridge, UK, 2009

21. H. Urkowitz, Energy detection of unknown deterministic signals, Proc. of the IEEE, vol. 55, no. 4, pp. 523531, Apr. 1967

22. E. Axell, G. Leus, E. G. Larsson, and H. V. Poor, Spectrum Sensing for Cognitive Radio: State-of-the-Art and Recent Advances, IEEE Sig. Proc. Mag., vol. 29, no. 3, pp. 101116, Mat 2012.

23. D. Cabric, S. M. Mishra, and R. W. Brodersen, Implementation issues in spectrum sensing for cognitive radios, Proc. of the 38th Asilomar Conference on Signals, Systems and Computers, pp. 772776, Nov. 2004.

24. H. Kim and K. G. Shin, In-Band Spectrum Sensing in IEEE 802.22 WRANs for Incumbent Protection, IEEE Trans. on Mob. Comp., vol. 9, no. 12, pp. 17661779, Dec. 2012.

25. D.Pauluzzi and N.Beaulieu, A comparison of SNR estimation techniques for the AWGN channel, Communications, IEEE Transactions on, vol. 48, no. 10, pp. 16811691, Oct 2000.

26. S. Cioni, G. Corazza, and M. Bousquet, An analytical characterization of maximum likelihood signal-to-noise ratio estimation, Wireless Communication Systems, 2005. 2nd International Symposium on, pp. 827830, Sept. 2005.

27. ETSI EN 302307 v1.3.1, Digital Video Broadcasting (DVB): Second Generation Framing Structure, Channel Coding and Modulation Systems for Broadcasting, Interactive Services, News Gathering and Other Broadband Satellite Applications (DVB-S2), Mar. 2013.

28. V. Icolari, A. Guidotti, D. Tarchi, and A. Vanelli-Coralli, An Interference Estimation Technique for Satellite Cognitive Radio Systems, to appear in ICC 2015, Jun. 2015

29. NASA, Socioeconomic Data and Applications Center (SEDAC), (accessed October 27, 2014). [Online]. Available: fhttp://sedac.ciesin.columbia.edug

30. S.K. Sharma, E. Lagunas, S. Maleki, S. Chatzinotas, J. Grotz, J. Krause, B. Ottersten, Resource Allocation for Cognitive Satellite Communications in Ka-band (17.7-19.7 GHz), Workshop on Cognitive Radios and Networks for Spectrum Coexistence of Satellite and Terrestrial Systems, IEEE Int. Conf. On Communications (ICC), London, UK, Jun 2015.

31. E. Lagunas, S.K. Sharma, S. Maleki, S. Chatzinotas, J. Grotz, J. Krause, B. Ottersten, Resource Allocation for Cognitive Satellite Uplink and Fixed-Service Terrestrial Coexistence in Ka-band, International Conference on Cognitive Radio Oriented Wireless Networks (CROWNCOM), Doha, Qatar, Apr 2015. 